\title{
Developing Plasmodium falciparum malaria vaccines for populations living in areas with stable parasite transmission
}

\author{
Lars Hviid* \\ Department of Infectious Diseases M7641, \\ Rigshospitalet, Blegdamsvej 9, \\ Copenhagen $\varnothing 2100$, Denmark \\ Fax: +45-35-45-76-44 \\ E-mail: lhcmp@rh.dk \\ ${ }^{*}$ Corresponding author

\section{Thor G. Theander} \\ Institute for Medical Microbiology and Immunology, \\ University of Copenhagen, \\ Blegdamsvej 3, Copenhagen N 2200, Denmark \\ Fax: +45-35-32-78-51 \\ E-mail: Theander@cmp.dk
}

\begin{abstract}
Individuals living in areas with stable transmission of Plasmodium falciparum parasites develop substantial protective immunity to the disease during childhood. Because of naturally acquired immunity, which appears mainly to target parasite-encoded Variable Surface Antigens (VSA) on the Infected Erythrocytes (IE), severe and life-threatening disease among adults in such areas is rare. However, low-grade asymptomatic parasitaemia continues to be present in a large proportion of people. So far, experimental P. falciparum malaria vaccination employing non-VSA antigens have resulted in variable degrees of protection, including sterile protection, but the duration of the protection afforded is short-lived, probably due to insufficient boosting. Based on these findings, our approach to vaccine development is to accelerate naturally acquired VSA-specific immunity. The ambition is to develop vaccines that will protect against mortality and severe morbidity, but which allow persistence of low-grade, asymptomatic infection. Hopefully, this approach will ensure regular boosting of immunity that appears necessary for the long-lasting protection required of vaccines to be deployed in malaria-endemic areas.
\end{abstract}

Keywords: Malaria; Plasmodium falciparum; immunity; vaccine; endemic countries; children; pregnant women; asexual blood stages.

Reference to this paper should be made as follows: Hviid, L. and Theander, T.G. (2007) 'Developing Plasmodium falciparum malaria vaccines for populations living in areas with stable parasite transmission', Int. J. Biotechnology, Vol. 9, Nos. 3/4, pp.292-300.

Biographical notes: Lars Hviid (MSc, PhD and DSc) is the Head of the malaria research laboratory in the Department of Infectious Diseases at Rigshospitalet, the main teaching hospital attached to the Medical School at University of Copenhagen, Denmark. He has been involved in malaria research 
since 1985. Most of his research has been conducted in close collaboration with colleagues in Africa, mainly in Sudan and Ghana. He is a member of the council of the Federation of the African Immunological Societies (http://www. faisoc.org) and serves on the editorial boards of Infection and Immunity and Trends in Parasitology.

Thor G. Theander (MD and DSc) is a Professor of Parasitology and International Health at the Faculty of Health Sciences, University of Copenhagen. He has been involved in malaria and leishmaniasis research since 1981. Most of his research has been conducted in a close collaboration with colleagues in Africa, mainly in Sudan and Tanzania. He is the Head of the Graduate School of International Health at University of Copenhagen.

"The road toward a safe and efficient malaria vaccine being available and usable on a large scale... will be long and chaotic" (Van de Perre and Dedet, 2004).

\section{Introduction}

About $40 \%$ of the world's population lives in areas with risk of being infected by Plasmodium falciparum parasites, the causative agent of the most severe form of malaria affecting humans. Falciparum malaria is estimated to cause 500 million clinical cases annually, mostly small children and pregnant women in sub-Saharan Africa, and to cost the lives of 1-3 million people. No vaccine suitable for mass vaccination is available despite decades of research (Richie and Saul, 2002). In the meantime, the malaria problem is growing worse, particularly in sub-Saharan Africa where it has always been the worst (Korenromp et al., 2003). This is mainly due to increasing problems with drug resistance, insecticide resistance and overwhelmed and underfunded health systems in most of the affected countries; problems that until recently have failed to attract the international attention they so glaringly deserve. Geo-political issues aside, there are two main types of problems with malaria: financial and scientific.

Malaria is a poverty trap. It is both a cause and a consequence of hardship, as has been eloquently and convincingly documented elsewhere (Sachs and Malaney, 2002). The bottom line is that the vast majority of malaria victims are poor or outright destitute. Accordingly, the incentives for pharmaceutical industry involvement in malaria research and development are limited, as the prospects for attractive returns on investments appear bleak (Ridley, 2002). In its place, a large proportion of the funding for research towards the development of new prophylactic and therapeutic interventions traditionally has come from military sources. Armed forces have a legitimate interest in protecting their personnel deployed in malarious areas - malaria casualties under such circumstances often outnumber those from combat - and their ambition is consequently to achieve complete protection of personnel in the short term (during deployment), while long-term sustainability of protection is of secondary concern. In contrast, for those living permanently in endemic areas the achievement of sustainable long-term protection is probably more important than solid protection. Encouragingly, there is a growing international awareness that the burden of malaria constitutes a major, intolerable obstacle to economic development in large parts of the world, and that there is a need to 
increase funding for both basic and applied research specifically addressing the problem of malaria facing people in endemic countries (http://www.rbm.who.int). This research includes malaria vaccine development.

Malaria vaccines have been on the research agenda for several decades. Twenty years ago, such vaccines appeared a feasible goal (Wernsdorfer, 1985) - and they still do (Targett, 2005). However, progress has been less than breath-taking and despite recent accomplishments (e.g. Alonso et al., 2004), truly effective vaccines suitable for mass vaccination in endemic countries remain roughly as far away as they were thought to be twenty or more years ago (Richie and Saul, 2002; Targett, 2005). Arguably, the main obstacle to malaria vaccine development has been - and to some extent continues to be - our lack of understanding of how immunity to malaria operates. Too often, antigens have been considered important mainly because they are known, and responses considered important mostly because they have been measured. Furthermore, associations have been often presented as proof of causal relationships.

At the Centre for Medical Parasitology, we have used as our starting point the fact that people living in areas of stable P. falciparum transmission acquire substantial protective immunity following years of exposure and repeated disease episodes. Protective immunity can therefore be acquired following exposure to P. falciparum parasites. We are studying the mechanisms and targets of this immunity with the ambition to learn how to accelerate its development through vaccination. The goal is vaccines that will provide long-lasting, sustainable protection from mortality and severe morbidity associated with specific P. falciparum malaria syndromes, but which allows continued presence of parasitemia. Vaccines that are specifically designed for peoples living in endemic countries.

\section{The $P$. falciparum parasites and the disease they cause}

Human infection with $P$. falciparum parasites begins with the injection of sporozoite-stage parasites during the blood meal of an infected Anopheles mosquito. The sporozoites rapidly migrate to the liver, where they invade hepatocytes. Once inside a liver cell, the parasite undergoes a series of nuclear divisions (known as exoerythrocytic or tissue schizogony) over about a week, which result in the formation of as many as 30,000 merozoites per sporozoite. This part of the infection is clinically silent. Eventually, the infected liver cells rupture and release the merozoites into the blood stream, where they invade erythrocytes and initiate the asexual multiplication cycle that is associated with all clinical symptoms of infection. Upon invasion, each merozoite transforms into a ring-stage parasite and after about $18 \mathrm{hr}$ into a trophozoite. The trophozoite undergoes several nuclear divisions resulting in the formation of a schizont composed of 8-24 merozoites. Forty-eight hours after invasion, the Infected Erythrocytes (IE) burst. The released merozoites invade new erythrocytes to continue the multiplication cycle. Many of the clinical symptoms of malaria are associated with IE rupture, and as infections are often more or less synchronous (all IE bursting at approximately the same time), symptoms may increase and decrease in a two-day cycle. At some point, some merozoites transform into male or female gametocytes upon erythrocyte invasion rather than continuing the asexual multiplication cycle. The gametocytes do not divide, and their further development depends on their ingestion by an Anopheles mosquito, inside which the parasite life cycle is completed. 
Only IE younger than about $18 \mathrm{hr}$ are present in the peripheral blood stream. Later developmental stages (trophozoites and schizonts) are retained in the vasculature of various tissues by means of parasite-encoded adhesion molecules on the IE surface. This process, called sequestration, is considered an important mechanism by which the parasite avoids recognition and destruction of IE in the spleen. While most malaria episodes in endemic areas are uncomplicated, severe complications such as cerebral malaria or severe malarial anaemia occurs in a minority of cases (Greenwood et al., 1991). Sequestration of IE at critical anatomical sites, such as the brain, appears to be involved in the pathogenesis of severe complications, such as cerebral malaria (Beeson and Brown, 2002). Each parasite has the ability to express a range of adhesins on the IE surface with specificity for a range of host receptors, presumably including brain-specific receptors, and can switch between expression of these adhesins by mechanisms that are currently being unravelled (Duraisingh et al., 2005; Freitas-Junior et al., 2005a,b). It thus appears that all parasites have the capacity to cause both uncomplicated and Severe Malaria (SM). We are now beginning to understand what determines which molecules are expressed on the IE surface and why only a minority of infections cause severe disease.

\section{Acquisition of protective immunity to $P$. falciparum malaria in areas with stable parasite transmission}

In areas with stable $P$. falciparum transmission, malaria mortality and severe morbidity is markedly concentrated among young children (typically those less than five years of age, but depending on endemicity). Older children continue to suffer clinical episodes, but little morbidity remains in adults. Nevertheless, most of the residents of such areas probably continue to have episodes of patent parasitemia throughout life. This is generally taken as evidence that protective immunity to malaria can be acquired in response to infection, but it also shows that the rate of acquisition is excruciatingly slow. Naturally acquired protective immunity appears to be mediated by $\operatorname{IgG}$, as passive transfer of IgG from adults effectively controls parasitemia and symptoms in children with P. falciparum malaria (Cohen et al., 1961; Sabchareon et al., 1991). Furthermore, the passive transfer experiments point to the asexual blood stages of the infection as the most important target. Specifically, a rapidly growing body of evidence points to the above-mentioned parasite-encoded adhesins on the IE surface as the main antigenic target of protective IgG (Bull et al., 1998; Nielsen et al., 2002; Ofori et al., 2002), making them attractive vaccine candidates. A major obstacle, however, is the fact that they undergo clonal antigenic variation (and are therefore generally known as Variant Surface Antigens (VSA), and that the VSA repertoire of individual parasite clones is different, making the global VSA repertoire potentially vast. Thus, if the goal is to develop a vaccine that will prevent any parasitemia, this complication probably precludes VSA as vaccine targets. However, if the goal is vaccines that will protect from mortality and severe morbidity from well-defined clinical syndromes - and for us, it is - this problem may be much less serious for two reasons.

Firstly, it has been repeatedly observed that, although sterile immunity is probably never achieved following exposure to $P$. falciparum parasites in endemic areas and acquisition of immunity to uncomplicated disease is slow, immunity to severe disease is acquired relatively quickly (Gupta et al., 1999). Pregnancy-Associated Malaria (PAM), 
which is associated with severe mother/child morbidity, is a particularly striking example of rapid development of protective immunity to a particular $P$. falciparum malaria syndrome after very few - perhaps a single - episode (Brabin, 1983).

Secondly, and very importantly, the pathogenesis of both PAM and SM in children have been associated with expression of particular VSA (VSA $\mathrm{PAM}_{\text {and }} \mathrm{VSA}_{\mathrm{SM}}$, respectively) with specific antigenic and functional properties (Beeson et al., 1999; Bull et al., 2000; Fried and Duffy, 1996; Nielsen et al., 2002; Ricke et al., 2000). VSA-specific immune responses appear to be the main factor determining which VSA will be expressed in a given infection, and therefore what the clinical consequences of it are likely to be. In the absence of specific immunity, parasites expressing virulent VSA $\left(\mathrm{VSA}_{\mathrm{sM}}\right)$ seem at a competitive advantage (probably because they mediate particularly efficacious IE adhesion) over parasites expressing non-SM VSA. VSA ${ }_{\mathrm{SM}}$-expressing parasites will thus dominate infections in non-immune individuals, and such infections will tend to be severe. As $\mathrm{VSA}_{\mathrm{SM}}$-specific immunity is acquired, this advantage gradually disappears and infections will be dominated by parasites expressing less and less virulent VSA. Detailed discussions of the evidence are available elsewhere (Hviid, 2004; Hviid, 2005; Hviid and Staalsoe, 2004).

\section{Identification of protective antigens}

The most rapid progress towards identifying VSA with potential as vaccine components has been related to studies of PAM. It illustrates well the power of advanced laboratory investigations guided by immuno-epidemiological field data (evidence-based vaccine research; Staalsoe et al., 2002). PAM constitutes an important exception to the general rule of malaria as a childhood disease in highly endemic areas, as previously protected women in such areas suddenly become susceptible to $P$. falciparum infection when becoming pregnant (McGregor and Smith, 1952). This susceptibility is very parity-dependent: while primigravidae are highly susceptible, women of high parity are largely protected (Brabin, 1983). From this type of evidence it became apparent that PAM is caused by parasites that do not thrive in non-pregnant hosts that and express antigens rapidly inducing protective immunity (McGregor, 1987). It is now firmly established that parasites causing PAM express particular VSA $\left(\mathrm{VSA}_{\mathrm{PAM}}\right)$ that are functionally and immunologically distinct from all other VSA (Fried and Duffy, 1996; Ricke et al., 2000). The fact that $P$. falciparum exposed men never possess $\mathrm{VSA}_{\mathrm{PAM}^{-}}$-specific IgG (Beeson et al., 1999; Ricke et al., 2000) and the rapid clearance of pregnancy-associated parasitemia post partum (Nguyen-Dinh et al., 1988) are strong evidence that parasites expressing $\mathrm{VSA}_{\mathrm{PAM}}$ cannot survive in non-pregnant hosts. Importantly, protection from the main adverse clinical consequences of PAM (prematurity, maternal anaemia and low birth weight of offspring) is mediated by VSA $_{\mathrm{PAM}}$-specific IgG (Duffy and Fried, 2003; Staalsoe et al., 2004). Expression of $\mathrm{VSA}_{\mathrm{PAM}}$ can be induced in vitro by selection for IE adhesion to the placental receptor Chondroitin Sulphate A (CSA) (Ricke et al., 2000). We used this method to enforce $\mathrm{VSA}_{\mathrm{PAM}}$ expression in the P. falciparum line NF54 (Delemarre and Van der Kaay, 1979), which is the parental line of clone 3D7 (Walliker et al., 1987). The 3D7 clone has had its entire genome sequenced and annotated (Gardner et al. 2002), and we could thus design specific primers for each of the 3D7 var genes encoding the main VSA family, called PfEMP1, and analyse changes in transcription of each var gene in response to selection 


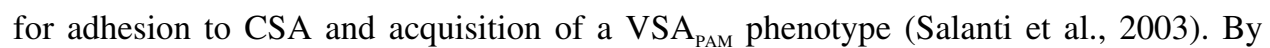
quantitative real-time PCR we could show that acquisition of $\mathrm{VSA}_{\mathrm{PAM}}$ expression was associated with markedly increased transcription of a single var gene, var2csa, and that this gene is preferentially transcribed by placental parasite isolates (Salanti et al., 2003; Tuikue-Ndam et al., 2005). Furthermore, the product of this gene, var2csa, is selectively expressed on the surface of $\mathrm{VSA}_{\mathrm{PAM}}$-expressing parasites, and VAR2CSA-specific antibodies are associated with a decreased risk of delivering low birth weight babies (Salanti et al., 2004). Recently, studies of parasites where the var2csa gene has been knocked out have shown that such parasites loose their ability to adhere to CSA and to acquire the $\mathrm{VSA}_{\mathrm{PAM}}$ phenotype, lending further support for the central involvement of VAR2CSA in the pathogenesis of PAM (Viebig et al., 2005). Very recent data from our laboratory indicate that VAR2CSA is indeed the main target of naturally acquired protective immunity to PAM, and that human VAR2CSA-specific IgG can inhibit IE adhesion to CSA in vitro. These findings, and the fact that the structure of the VAR2CSA gene is both different from all other var genes (supporting the antigenic uniqueness of VAR2CSA) and conserved between genetically distinct isolates (Gardner et al., 2002; Salanti et al., 2003), have made VAR2CSA the current leading candidate for development of a PAM-specific vaccine (Smith and Deitsch, 2004).

In a parallel approach, we are attempting to identify VSA that are associated with the pathogenesis of SM in children. Previous studies have shown that such VSA (VSA $\left.{ }_{\mathrm{SM}}\right)$ are serologically and functionally distinct from VSA associated with Uncomplicated Malaria (UM) (Bull et al., 2000; Nielsen et al., 2002). We have developed in vitro selection protocols to induce expression of $\mathrm{VSA}_{\mathrm{SM}}$ (Staalsoe et al., 2003) and used such parasites to investigate which var genes are affected by selection for $\mathrm{VSA}_{\mathrm{SM}}$ expression (Jensen et al., 2004). These studies revealed that selection for $\mathrm{VSA}_{\mathrm{SM}}$ expression is associated with preferential expression of a particular, structurally related subset of var genes that we have called Group A (Jensen et al., 2004; Kraemer and Smith, 2003; Lavstsen et al., 2003). A major challenge immediately ahead will be an establishment of the receptor specificity of $\mathrm{VSA}_{\mathrm{SM}}$. This is likely to be more difficult than for $\mathrm{VSA}_{\mathrm{PAM}}$ since the pathogenesis of SM is multifactorial, and since multiple receptors are undoubtedly involved, even for individual types of VSA (Chen et al., 2000).

\section{Concluding remarks}

Complete, strain-transcending prevention of parasitemia and long-lasting (years to decades) protection are often cited as the main virtues of an ideal malaria vaccine. No such vaccine exists. We consider it questionable if ever it will. However, nature has shown that non-sterilising, long-lasting immunity protecting against mortality and severe morbidity can be developed. Maintenance of the protection afforded appears to require regular presence of low-grade parasitemia, and asymptomatic parasitemia has been shown to decrease the risk of subsequent clinical attacks (Ofori et al., 2002; Gaye et al., 2005). Naturally acquired protection from malaria rests on VSA-specific IgG. Preservation of this immunity - and of immunity against particular VSA artificially induced by vaccination - thus appears to require repeated probing and boosting of VSA-specific immunity by the infecting parasites. This may render VSA-based vaccines inadequate for short-term visitors to endemic areas (e.g. tourists and military personnel). However, they would probably be acceptable - indeed, they may be preferable (because 
of the likelihood of long-term protection) - to people with permanent residence in areas with stable parasite transmission. Such vaccines, designed specifically for developing countries, is our goal.

\section{Acknowledgements}

We gratefully acknowledge the indispensable contributions of our colleagues at CMP and our collaborators in Africa and elsewhere. We also gratefully acknowledge the people who all have volunteered to participate as research subjects. Our research receives support from many sources, but the Bill and Melinda Gates Foundation, the Commission of the European Communities, the Danish Medical Research Council, and last but not the least, the Danish Research Council for Development Research deserve particular mention.

\section{References}

Alonso, P., et al. (2004) 'Efficacy of the RTS, S/AS02A vaccine against Plasmodium falciparum infection and disease in young African children: randomised controlled trial', Lancet, Vol. 364, pp.1411-1420.

Beeson, J.G. and Brown, G.V. (2002) 'Pathogenesis of Plasmodium falciparum malaria: the roles of parasite adhesion and antigenic variation', Cellular and Molecular Life Sciences, Vol. 59, pp.258-271.

Beeson, J.G., Brown, G.V., Molyneux, M.E., Mhango, C., Dzinjalamala, F. and Rogerson, S.J. (1999) 'Plasmodium falciparum isolates from infected pregnant women and children are associated with distinct adhesive and antigenic properties', Journal of Infectious Diseases, Vol. 180, pp.464-472.

Brabin, B.J. (1983) 'An analysis of malaria in pregnancy in Africa', Bulletin World Health Organisation, Vol. 61, pp.1005-1016.

Bull, P.C., Kortok, M., Kai, O., Ndungu, F., Ross, A., Lowe, B.S., Newbold, C.I. and Marsh, K. (2000) 'Plasmodium falciparum-infected erythrocytes: agglutination by diverse Kenyan plasma is associated with severe disease and young host age', Journal of Infectious Diseases, Vol. 182, pp.252-259.

Bull, P.C., Lowe, B.S., Kortok, M., Molyneux, C.S., Newbold, C.I. and Marsh, K. (1998) 'Parasite antigens on the infected red cell are targets for naturally acquired immunity to malaria', Nature Medicine, Vol. 4, pp.358-360.

Chen, Q.J., Heddini, A., Barragan, A., Fernandez, V., Pearce, S.F.A. and Wahlgren, M. (2000) 'The semiconserved head structure of Plasmodium falciparum erythrocyte membrane protein 1 mediates binding to multiple independent host receptors', Journal of Experimental Medicine, Vol. 192, pp.1-9.

Cohen, S., McGregor, I.A. and Carrington, S. (1961) 'Gammaglobulin and acquired immunity to human malaria', Nature, Vol. 192, pp.733-737.

Delemarre, B.J. and Van der Kaay, H.J. (1979) 'Malaria tropica op natuurlijke wijze verkregen in Nederland', Nederlands Tijdschrift Geneeskunde, Vol. 123, pp.1981-1982.

Duffy, P.E. and Fried, M. (2003) 'Antibodies that inhibit Plasmodium falciparum adhesion to chondroitin sulfate A are associated with increased birth weight and the gestational age of newborns', Infection and Immunity, Vol. 71, pp.6620-6623.

Duraisingh, M.T., Voss, T.S., Marty, A.J., Duffy, M.F., Good, R.T., Thompson, J.K., Freitas-Junior, L.H., Scherf, A., Crabb, B.S. and Cowman, A.F. (2005a) 'Heterochromatin silencing and locus repositioning linked to regulation of virulence genes in Plasmodium falciparum', Cell, Vol. 121, pp.13-24. 
Freitas-Junior, L.H., Hernandez-Rivas, R., Ralph, S.A., Montiel-Condado, D., Ruvalcaba-Salazar, O.K., Rojas-Meza, A.P., Mancio-Silva, L., Leal-Silvestre, R.J., Gontijo, A.M., Shorte, S. and Scherf, A. (2005b) 'Telomeric heterochromatin propagation and histone acetylation control mutually exclusive expression of antigenic variation genes in malaria parasites', Cell, Vol.121, pp.25-36.

Fried, M. and Duffy, P.E. (1996) 'Adherence of Plasmodium falciparum to chondroitin sulphate A in the human placenta', Science, Vol. 272, pp.1502-1504.

Gardner, M.J., et al. (2002) 'Genome sequence of the human malaria parasite Plasmodium falciparum', Nature, Vol. 419, pp.498-511.

Gaye, O. et al. (2005) Abstract O-310, Medicine and Health in the Tropics, Marseille, France, 11-15 September.

Greenwood, B.M., Marsh, K. and Snow, R. (1991) 'Why do some children develop severe malaria?' Parasitology Today, Vol. 7, pp.277-281.

Gupta, S., Snow, R.W., Donnelly, C.A., Marsh, K. and Newbold, C. (1999) 'Immunity to non-cerebral severe malaria is acquired after one or two infections', Nature Medicine, Vol. 5, pp.340-343.

Hviid, L. (2004) 'The immuno-epidemiology of pregnancy-associated malaria: a variant surface antigen-specific perspective', Parasite Immunology, Vol. 26, pp.477-486.

Hviid, L. (2005) 'Naturally acquired immunity to Plasmodium falciparum malaria in Africa', Acta Tropica, Vol. 95, pp.270-275.

Hviid, L. and Staalsoe, T. (2004) 'Malaria immunity in infants: a special case of a general phenomenon?' Trends in Parasitology, Vol. 20, pp.66-72.

Jensen, A.T.R., et al. (2004) 'Plasmodium falciparum associated with severe childhood malaria preferentially expresses PfEMP1 encoded by Group A var genes', Journal of Experimental Medicine, Vol. 199, pp.1179-1190.

Korenromp, E.L., Williams, B.G., Gouws, E., Dye, C. and Snow, R.W. (2003) 'Measurement of trends in childhood malaria mortality in Africa: an assessment of progress toward targets based on verbal autopsy', Lancet Infectious Diseases, Vol. 3, pp.349-358.

Kraemer, S.M. and Smith, J.D. (2003) 'Evidence for the importance of genetic structuring to the structural and functional specialization of the Plasmodium falciparum var gene family', Molecular Microbiology, Vol. 50, pp.1527-1538.

Lavstsen, T., Salanti, A., Jensen, A.T.R., Arnot, D.E. and Theander, T.G. (2003) 'Sub-grouping of Plasmodium falciparum 3D7 var genes based on sequence analysis of coding and non-coding regions', Malariology Journal, Vol. 2, p.27.

McGregor, I.A. (1987) 'Thoughts on malaria in pregnancy with consideration of some factors which influence remedial strategies', Parassitologia, Vol. 29, pp.153-163.

McGregor, I.A. and Smith, D.A. (1952) 'A health, nutrition and parasitological survey in a rural village (Keneba) in west Kiang, Gambia', Transactions of the Royal Society Tropical Medicine and Hygiene, Vol. 46, pp.403-427.

Nguyen-Dinh, P., Steketee, R.W., Greenberg, A.E., Wirima, J.J., Mulenda, O. and Williams, S.B. (1988) 'Rapid spontaneous postpartum clearance of Plasmodium falciparum parasitaemia in African women', Lancet, Vol. 2, pp.751-752.

Nielsen, M.A., Staalsoe, T., Kurtzhals, J.A.L., Goka, B.Q., Dodoo, D., Alifrangis, M., Theander, T.G., Akanmori, B.D. and Hviid, L. (2002) 'Plasmodium falciparum variant surface antigen expression varies between isolates causing severe and non-severe malaria and is modified by acquired immunity', Journal of Immunology, Vol. 168, pp.3444-3450.

Ofori, M.F., Dodoo, D., Staalsoe, T., Kurtzhals, J.A.L., Koram, K., Theander, T.G., Akanmori, B.D. and Hviid, L. (2002) 'Malaria-induced acquisition of antibodies to Plasmodium falciparum variant surface antigens', Infection and Immunity, Vol. 70, pp.2982-2988.

Richie, T.L. and Saul, A. (2002) 'Progress and challenges for malaria vaccines', Nature, Vol. 415, pp.694-701. 
Ricke, C.H., Staalsoe, T., Koram, K., Akanmori, B.D., Riley, E.M., Theander, T.G. and Hviid, L. (2000) 'Plasma antibodies from malaria-exposed pregnant women recognize variant surface antigens on Plasmodium falciparum-infected erythrocytes in a parity-dependent manner and block parasite adhesion to chondroitin sulphate A', Journal of Immunology, Vol. 165, pp.3309-3316.

Ridley, R.G. (2002) 'Medical need, scientific opportunity and the drive for antimalarial drugs', Nature, Vol. 415, pp.686-693.

Sabchareon, A., Burnouf, T., Ouattara, D., Attanath, P., Bouharoun, T.H., Chantavanich, P., Foucault, C., Chongsuphajaisiddhi, T. and Druilhe, P. (1991) 'Parasitologic and clinical human response to immunoglobulin administration in falciparum malaria', American Journal of Tropical Medicine and Hygiene, Vol. 45, pp.297-308.

Sachs, J. and Malaney, P. (2002) 'The economic and social burden of malaria', Nature, Vol. 415, pp.680-685.

Salanti, A., Dahlbäck, M., Turner, L., Nielsen, M.A., Barfod, L., Magistrado, P., Jensen, A.T.R., Lavstsen, T., Ofori, M.F., Marsh, K., Hviid, L. and Theander, T.G. (2004) 'Evidence for the involvement of VAR2CSA in pregnancy-associated malaria', Journal of Experimental Medicine, Vol. 200, pp.1197-1203.

Salanti, A., Staalsoe, T., Lavstsen, T., Jensen, A.T.R., Sowa, M.P.K., Arnot, D.E., Hviid, L. and Theander, T.G. (2003) 'Selective upregulation of a single distinctly structured var gene in CSA-adhering Plasmodium falciparum involved in pregnancy-associated malaria', Molecular Microbiology, Vol. 49, pp.179-191.

Smith, J.D. and Deitsch, K.W. (2004) 'Pregnancy-associated malaria and the prospects for syndrome-specific antimalaria vaccines', Journal of Experimental Medicine, Vol. 200, pp.1093-1097.

Staalsoe, T., Jensen, A.T.R., Theander, T.G. and Hviid, L. (2002) 'Novel Plasmodium falciparum malaria vaccines: evidence-based searching for variant surface antigens as candidates for vaccination against pregnancy-associated malaria', Immunology Letters, Vol. 84, pp.133-136.

Staalsoe, T., Nielsen, M.A., Vestergaard, L.S., Jensen, A.T.R., Theander, T.G. and Hviid, L. (2003) 'In vitro selection of Plasmodium falciparum 3D7 for expression of variant surface antigens associated with severe malaria in African children', Parasite Immunology, Vol. 25, pp.421-427.

Staalsoe, T., Shulman, C.E., Bulmer, J.N., Kawuondo, K., Marsh, K. and Hviid, L. (2004) 'Variant surface antigen-specific IgG and protection against the clinical consequences of pregnancy-associated Plasmodium falciparum malaria', Lancet, Vol. 363, pp.283-289.

Targett, GA. 'Malaria vaccines 1985-2005: a full circle?' Trends in Parasitology, Vol. 21, pp. 499-503

Tuikue-Ndam, N.G., Salanti, A., Bertin, G., Dahlbäck, M., Fievet, N., Turner, L., Gaye, A., Theander, T.G. and Deloron, P. (2005) 'High level of var2csa transcription by Plasmodium falciparum isolated from the placenta', Journal of Infectious Diseases, Vol. 192, pp.331-335.

Van de Perre, P. and Dedet, J.P. (2004) 'Vaccine efficacy: winning a battle (not war) against malaria', Lancet, Vol. 364, pp.1380-1383.

Viebig, N.K., Gamain, B., Scheidig, C., Lepolard, C., Przyborski, J., Lanzer, M., Gysin, J. and Scherf, A. (2005) 'A single member of the Plasmodium falciparum var multigene family determines cytoadhesion to the placental receptor chondroitin sulphate A', EMBO Reports, Vol. 6, pp.1-7.

Walliker, D., Quakyi, I.A., Wellems, T.E., McCutchan, T.F., Szarfman, A., London, W.T., Corcoran, L.M., Burkot, T.R. and Carter, R. (1987) 'Genetic analysis of the human malaria parasite Plasmodium falciparum', Science, Vol. 236, pp.1661-1666.

Wernsdorfer, W.H. (1985) 'Towards a malaria vaccine - discussion', Parasitology Today, Vol. 1, pp.158-159. 